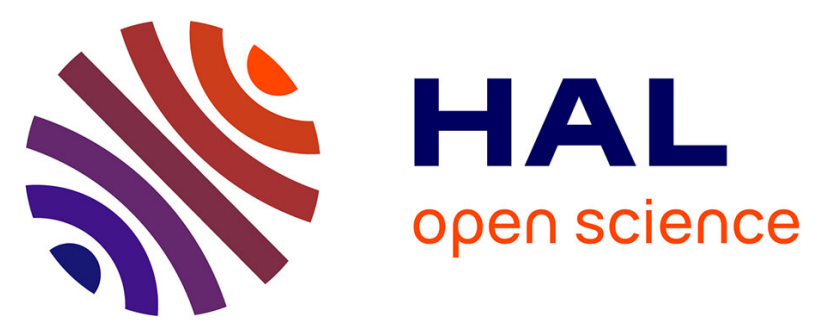

\title{
Impédance acoustique d'un liquide à la surface d'un quartz coupe AT
}

L. Tessier, M. Lethiecq, Dominique Certon, F. Patat

\section{To cite this version:}

L. Tessier, M. Lethiecq, Dominique Certon, F. Patat. Impédance acoustique d'un liquide à la surface d'un quartz coupe AT. Journal de Physique IV Proceedings, 1994, 04 (C5), pp.C5-1205-C5-1208. 10.1051/jp4:19945266 . jpa-00252956

\section{HAL Id: jpa-00252956 https://hal.science/jpa-00252956}

Submitted on 1 Jan 1994

HAL is a multi-disciplinary open access archive for the deposit and dissemination of scientific research documents, whether they are published or not. The documents may come from teaching and research institutions in France or abroad, or from public or private research centers.
L'archive ouverte pluridisciplinaire HAL, est destinée au dépôt et à la diffusion de documents scientifiques de niveau recherche, publiés ou non, émanant des établissements d'enseignement et de recherche français ou étrangers, des laboratoires publics ou privés. 


\title{
Impédance acoustique d'un liquide à la surface d'un quartz coupe AT
}

\author{
L. TESSIER, M. LETHIECQ, D. CERTON et F. PATAT
}

GIP Ultrasons, Faculté de Médecine, Laboratoire de Biophysique Médicale, Bâtiment Vialle, 2 bis boulevard Tonnellé, BP. 3223, 37032 Tours cedex, France

\begin{abstract}
The Shear acoustic impedance of a liquid can be determined from the complex reflection coefficient of the acoustic wave incident on the AT-cut quartz/liquid interface. This complex acoustic coefficient is related to the down shift of the resonant frequency as well as the decrease of the quality factor, due to the liquid load. The effective acoustic impedance measured with the thickness shear mode resonator is shown to be composed of three main components: a preponderant complex component due to viscous coupling between the quartz and the liquid, a pure imaginary additional component due to liquid trapped in the surface irregularities and a pure real additional component due to compressional wave generation in the bulk liquid.
\end{abstract}

\section{INTRODUCTION}

Un quartz de coupe AT, vibrant selon le mode de cisaillement en épaisseur, constitue un excellent résonateur piézo-électrique. En effet son coefficient en température est nul à température ambiante et son coefficient de surtension est généralement supérieur à 105. Par ailleurs, en raison de leur grande sensibilité aux contraintes mécaniques, ces résonateurs sont utilisés depuis longtemps comme capteurs gravimétriques. Les applications en phase gazeuse sont principalement le contrôle de dépôt sous vide de film en couche mince et la détection de gaz en chimie. Le seuil de résolution d'une microbalance à quartz dépend de sa fréquence de fonctionnement et de l'acuité du résonateur, typiquement on atteint une résolution de l'ordre du $\mathrm{ng} / \mathrm{cm}^{2}$. Les microbalances à quartz peuvent également fonctionner en solution; elles sont principalement utilisées en électrochimie pour l'analyse gravimétrique de réactions d'oxydoréduction [1-2]. Depuis unedizaine d'années, les résonateurs de quartz AT se sont avérés des capteurs de choix pour mesurer l'impédance acoustique transverse d'un film solide ou liquide, déposé à sa surface [3-5]. Nous présentons ici, le principe de la mesure de l'impédance acoustique d'un liquide Newtonien et l'analyse des différents mécanismes de couplage quartz/liquide mis en évidence expérimentalement.

\section{PRINCIPE PHYSIQUE ET DISPOSITIF EXPÉRIMENTAL}

Le résonateur de quartz AT se caractérise par sa fréquence de résonance $\mathrm{f}_{\mathrm{n}}$ et son coefficient de surtension $Q_{n}$ ( $n$ étant le rang de l'harmonique). Ces deux caractéristiques de résonance sont très sensibles aux contraintes imposées par le milieu extérieur. Les contraintes imposées par un liquide à la surface du quartz sont principalement dues à la viscosité. Le couplage quartz/liquide qui en résulte se traduit par le rayonnement dans le liquide d'une onde acoustique transversale fortement amortie. Le coefficient de réflexion acoustique à l'interface quartz/liquide s'écrit,

$$
r=\frac{Z_{q}-Z_{l}}{Z_{q}+Z_{l}} \approx 1-2 \frac{Z_{l}}{Z_{q}} ; \quad \text { si }\left|Z_{l}\right| \ll Z_{q}
$$


où $Z_{l}=R+j X$, est l'impédance acoustique transverse du liquide, $Z_{q}=\rho_{q} c_{q}$, l'impédance acoustique du quartz, $\rho_{q}$ et $c_{q}$ étant respectivement la densité et la célérité de l'onde acoustique transverse. Le coefficient $r$ permet de déterminer, à l'interface quartz/liquide, le déphasage et l'énergie transmise en fonction des impédances $Z_{l}$ et $Z_{q}$, ce qui finalement nous amène aux expressions qui relient les variations de $f_{n}$ et $Q_{n}$ en fonction de limpédance acoustique transverse du liquide:

$$
\frac{\Delta \mathrm{f}_{\mathrm{n}}}{\mathrm{f}_{\mathrm{n}}}=-\frac{\mathrm{X}}{\mathrm{n} \pi \mathrm{Z}_{\mathrm{q}}} \quad \text { et } \quad \Delta\left(Q_{n}^{-1}\right)=\frac{2 R}{n \pi Z_{q}}
$$

Les mesures successives de $f_{n}$ et $Q_{n}$ pour le quartz sec puis mouillé permettent donc de déterminer expérimentalement les parties réelle et imaginaire de l'impédance acoustique transverse du liquide. Notons toutefois que l'impédance acoustique mesurée est l'impédance effective du liquide; cette mesure ne sera égale à la valeur théorique d'un liquide Newtonien que dans le cas où le couplage quartz/liquide est exclusivement de nature visqueuse. L'expression théorique de $Z_{l}$ pour un liquide Newtonien s'écrit,

$$
Z_{1}=\left(\pi f_{n} \rho_{1} \eta_{1}\right)^{1 / 2}(1+j) \quad ; \quad R=X=\left(\pi f_{n} \rho_{1} \eta_{1}\right)^{1 / 2}
$$

où $\eta l$ et $\rho l$ sont respectivement la viscosité et la densité du liquide. L'impédance transverse d'un liquide Newtonien se distingue par l'égalité de ses composantes réelle et imaginaire.

Le dispositif expérimental, pour la mesure des impédances acoustiques des liquides, est constitué principalement d'une cellule, permettant de mettre en contact le quartz et l'échantillon liquide, et d'un analyseur d'impédance mesurant le spectre de la conductance électrique autour de la résonance du mode de cisaillement en épaisseur. Un algorithme, ajustant la fonction Lorentzienne d'une résonance amortie avec les spectres expérimentaux, permet de déterminer la fréquence de résonance et le coefficient de surtension [6].

\section{RÉSULTATS ET DISCUSSION}

Dans le but d'étudier l'influence de la viscosité nous utilisons des mélanges eau/éthylène glycol à différentes concentrations $(0,10,20,40,60 \%$ d'éthylène glycol).

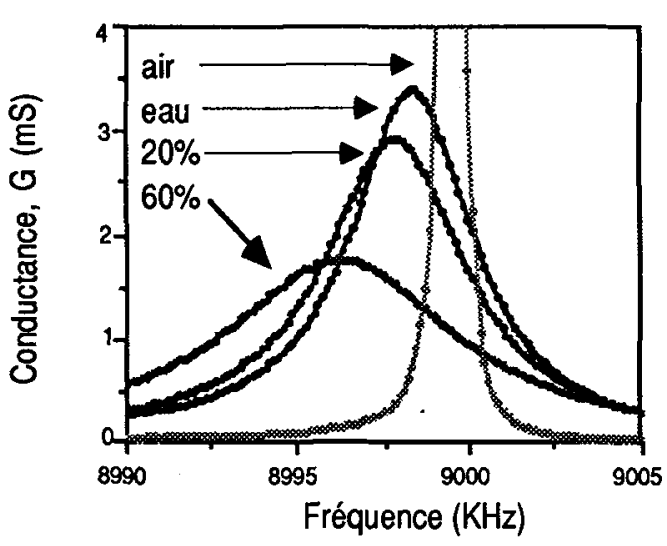

Fig. 1: Spectres de la conductance électrique du quartz chargé par des mélanges eauléthylène glycol.

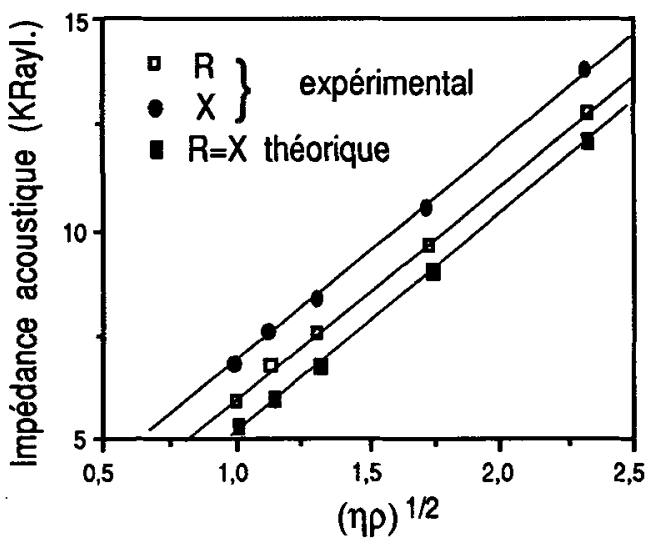

Fig.2: Parties réelle et imaginaire des impédances acoustiques en fonction de $\sqrt{\eta \rho}$.

On constate sur la figure 1 que plus le liquide est visqueux plus la résonance est amortie et plus le spectre se décale vers la gauche. Ainsi $Q_{n}$ et $f_{n}$ décroissent parallèlement lorsque la viscosité augmente, ce qui correspond également à une augmentation des parties imaginaire et réelle de l'impédance acoustique. 
La figure 2 montre que $R$ et $X$ varient linéairement en fonction de $\left(\eta_{1} \rho_{l}\right)^{1 / 2}$, ce qui est en accord avec l'expression théorique (3); en revanche on observe une partie imaginaire systématiquement supérieure à la partie réelle, elle même légèrement supérieure à l'impédance acoustique transverse d'un liquide Newtonien.

Afin de mettre en évidence l'influence de la rugosité de surface du quartz sur l'impédance acoustique effective, nous avons comparé les mesures obtenues avec deux quartz de rugosités nettement différentes (figure 3). Les mesures d'impédances ont été effectuées en fonction de la fréquence de fonctionnement du résonateur. Pour ce faire nous opérons aux harmoniques d'ordre supérieurs du mode de cisaillement en épaisseur. Nous avons observé une très nette surévaluation de la partie imaginaire de l'impédance mesurée avec le quartz le plus rugueux. La figure 4, nous montre l'excès de la composante imaginaire par rapport à la valeur théorique en fonction de la fréquence de fonctionnement.

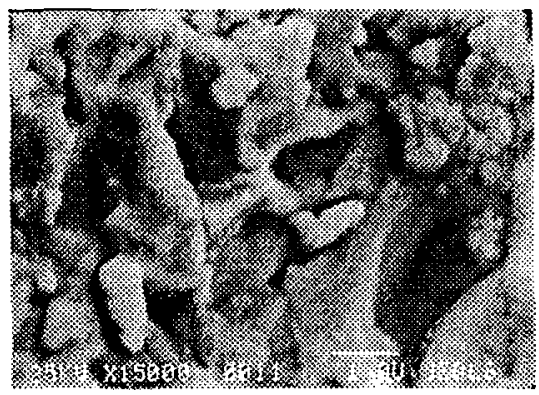

Fig. 3a: Quartz rugueux.

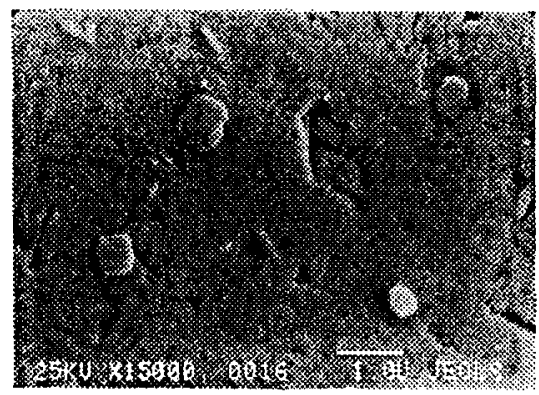

Fig. 3b: Quartz lisse.

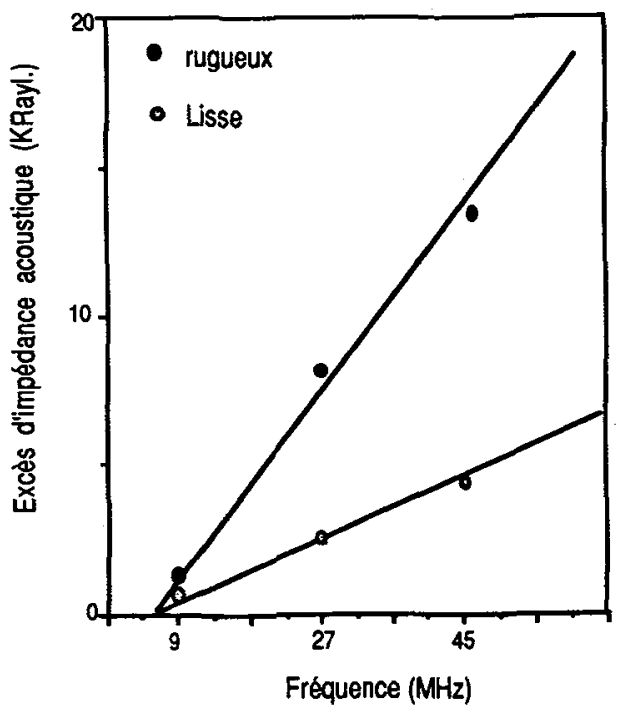

Fig. 4: Terme excédentaire de la partie imaginaire en fonction de la fréquence de fonctionnement du résonateur.

On observe d'une part que le terme imaginaire excédentaire est proportionnel à la fréquence de fonctionnement, et d'autre part que la pente des droites croit avec la taille des rugosités. Cette constatation suggère fortement que ce terme additionnel correspond au liquide piégé dans les anfractuosités de l'interface. L'impédance acoustique associée à ce phénomène est effectivement une impédance imaginaire pure, proportionnelle à la fréquence d'oscillation et à l'épaisseur moyenne, $d$, de la couche de liquide "rigidement" lié à la surface ( $d$ est fonction de la taille des rugosités). L'expression thérique de ce terme additionnel est donnée ci-dessous:

$$
Z_{l}^{r}=j \omega \rho_{l} d
$$

A l'aide de l'expression 4, on peut déduire de la pente des droites expérimentales, $Z_{1}^{r}(f)$, la valeur du terme imaginaire due à la rugosité pour chaque quartz utilisé. Compte tenu de la précision des mesures $(1-5 \%)$, les valeurs ainsi calculées sont en bon accord avec l'écart trouvé entre la partie imaginaire de l'impédance effective et celle de l'impédance d'un liquide Newtonien. En revanche le couplage rigide du liquide "interfacial" n'explique pas le léger excédant observé également pour la composante réelle de l'impédance acoustique effective. Cependant une étude en fonction de la température a montré que ce terme supplémentaire correspondait à une fraction d'énergie dissipée dans le liquide par le rayonnement d'ondes longitudinales [6]. 


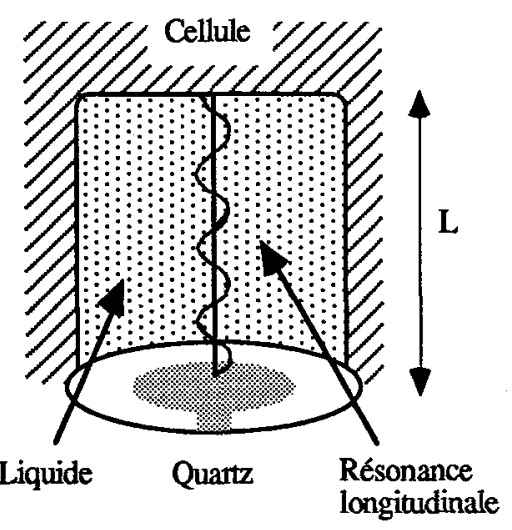

Fig. 5: Cellule de mesure (quartz + liquide)
En effet, les mesures d'impédance acoustique de différents liquides en fonction de la température ont permis de mettre en évidence l'existence de résonances harmoniques d'ondes longitudinales à l'intérieur de la cellule (figure 5). L'impédance acoustique associée à ce couplage longitudinal est donnée ci-dessous,

$$
Z_{1}^{p}=\varepsilon \bar{Z}_{1} \frac{\bar{Z}_{8}+\bar{Z}_{1} j \tan (k L)}{\bar{Z}_{1}+\bar{Z}_{z} j \tan (k L)}
$$

où $\bar{Z}_{1}$ et $\bar{Z}_{\mathbf{z}}$ sont respectivement les impédances longitudinales du liquide et du matériau de la cellule, $L$ est la hauteur de la cellule, $K$ est le nombre d'onde associé à l'onde longitudinale dans le liquide et $\varepsilon$ est un coefficient empirique de l'ordre de $2.10^{-4}$.

A température ambiante $Z_{1}^{p}$ s'est avérée avoir essentiellement une composante réelle proche du terme excédentaire mentionné auparavant.

\section{CONCLUSION}

En conclusion, on peut donc dire que l'impédance acoustique d'un liquide mesurée par un résonateur de quartz AT se compose de trois termes principaux: un terme complexe dû au couplage visqueux, un terme purement imaginaire dû au couplage rigide du liquide piégé dans les rugosités, et un terme complexe résultant d'un couplage longitudinal dû à la génération d'ondes longitudinales stationnaires. D'autre part ce travail montre que la mesure d'impédance acoustique par le résonateur de quartz AT est une technique efficace et relativement simple de mise en oeuvre; elle s'applique aussi bien à l'étude des propriétés physico-chimiques des interfaces solide/liquide [5, 7], qu'au développement de biocapteurs à base de quartz AT [8].

\section{RÉFÉRENCES}

[1] Schumacher R., Borges G., Kanazawa K. K., The quartz microbalance: a sensitive tool to probe surface reconstruction's on gold electrodes in liquid, Surf. Sci., vol. 163, 1985, pp. L621-L626.

[2] Beck R., Pitterman U., Weil K. G., Influence of the surface micro structure on the coupling between a quartz oscillator and a liquid, J. Electrochem. Soc., vol. 139, 1992, pp. 453-461.

[3] Benes E., Improved quartz crystal microbalance technique, J. Appl. Phys., vol. 56, 1984, pp. 608626.

[4] Wajid A., Improving the accuracy of a quartz crystal microbalance with automatic determination of acoustic impedance ratio, Rev. Sci. Instrum., vol. 62, 1991, pp. 2026-2030.

[5] Lea M. J., Fozooni P., The transverse acoustic impedance of an inhomogeneous viscous fluid, Ultrasonics, may, 1985, pp. 133-137.

[6] Tessier L., Immunodétection par un résonateur a quartz, 1993, Université de Tours.

[7] Martin S. J., Frye G. C., Ricco A. J., Effect of surface roughness on the response of thickness-shear mode resonators in liquids, Anal. Chem., vol. 65, 1993, pp. 2910-2922.

[8] Tessier L., Patat F., Schmitt N., Lethiecq M., Frangin Y., Guilloteau D., Significance of mass and viscous loads discrimination for an AT-quartz blood group immunosensor, à paraître dans Sensors and Actuators. $B$. 